Research Article

\title{
Robust Optimization Solution to Emergency Mobile Facility Fleet Size and Location
}

\author{
Jianxun Li $\mathbb{D}^{1},{ }^{1}$ Kin Keung Lai $\mathbb{D}^{2}{ }^{2}$ and Qiuping Lin $\mathbb{D}^{3}$ \\ ${ }^{1}$ School of Economics and Management, Xi'an University of Technology, Xi'an 710048, China \\ ${ }^{2}$ College of Economics, Shenzhen University, Shenzhen 518060, China \\ ${ }^{3}$ Herberger Business School, St. Cloud State University, St. Cloud, MN 56301, USA \\ Correspondence should be addressed to Kin Keung Lai; mskklai@outlook.com
}

Received 22 May 2019; Revised 26 August 2019; Accepted 6 November 2019; Published 22 November 2019

Academic Editor: A. M. Bastos Pereira

Copyright (C) 2019 Jianxun Li et al. This is an open access article distributed under the Creative Commons Attribution License, which permits unrestricted use, distribution, and reproduction in any medium, provided the original work is properly cited.

\begin{abstract}
Allocation of emergency mobile facility is the key problem of the emergency response system, which affects the cost and the satisfaction of services for emergency so as to rapidly respond to disasters, contagions, etc. In order to seek a reasonable fleet size and locations of emergency mobile facilities, we propose a two-stage programming model with the objective function of minimizing the total cost. With uncertain characteristics of emergency event, the model conforms to the requirement for noninterruptible service and tries to satisfy all emergency demands. For overcoming the computational difficulty of emergency mobile facility fleet size and location problem, a robust optimization approach and modified ant colony algorithm are employed to obtain solutions. The illustrative example shows that the model can provide a reasonable solution to the determination of the fleet size and locations of emergency mobile facilities and that the risk recognition factor of the model can further guide decisionmaking.
\end{abstract}

\section{Introduction}

The emergency mobile facility (EMF) refers to public facilities equipped for dealing with emergency events; it is usually a special vehicle which can flexibly change their position in certain range and provide responses to disasters, contagions, and other emergencies. At present, researchers have proposed the basic model of EMFs planning and analyzed the problems of routing, locating, scheduling, etc. However, existing studies mainly assume that the service for emergency can be decomposed by time. The service can be interrupted and divided into multiple time periods served with EMFs, reducing the practicability of the planning model. In general, most emergency events are uninterruptible and indivisible. For example, the life detection after disasters, the treatment on sudden infectious diseases, and the disposal of public safety events all require continuous services. Since EMFs usually have the same structure, equipment, and rescue method, if a service process is interrupted due to EMFs replacement, a large amount of time will be spent on the service equipment replacement and rescue message passing, thus losing the appropriate time to save lives and property. In addition, the available research on the fleet size and locations of EMFs only attributes the uncertainty of emergency events to the indeterminacy of demand. The uncertainty of occurrence location and wait time of emergency events lacks analysis. Therefore, in order to seek rational fleet size and locations of EMFs, we should completely consider the uncertainty of emergency events and the continuity of services. Furthermore, the construction of an EMF team involve not only maintenance cost, such as equipment cost, insurance cost, repair cost, and manpower cost but also time cost of travel time and service time. Thus, the objective function should minimize the total cost of emergency service (the service for emergency event).

Based on the abstract description of emergency event, this paper presents a model of EMF fleet size and location to minimize the total cost of service. The model takes the uninterrupted service as the fundamental constraint to try meeting all demands. After robust optimization of the 
uncertainty in the model, an ant colony algorithm with an ant team is put forward to obtain solutions. The results show that it is helpful to search a reasonable solution to the determination of the fleet size and locations of EMFs.

The remainder of the paper is organized as follows. Section 2 briefly reviews related work on emergency facilities location-routing problem and fleet sizing decision problem. Section 3 presents a fleet size and location model of EMF based on uncertain factors. In Section 4, the robust optimization of the model is presented and solved by ant colony algorithm. In Sections 5 and 6, we set Xianyang city, China, as an example and analyze the risk recognition factor of the model. Section 7 concludes the paper and provides remarks.

\section{Related Literature}

EMF is a special facility that can move flexibly among different locations to present radiation-type emergency services for surrounding areas. Due to the high service cost of emergency service by EMF, the related research focuses largely on selecting fleet size and allocating facilities reasonably. As early as in 1971, Toregas [1] analyzed the positioning of emergency services equipment, which aroused scholars' great attention, and soon it was introduced to location issue of disaster response [2] and emergency medical service [3]. For example, Döyen [4] put forward a facility location model for humanitarian relief logistics. An [5] proposed an emergency service facility location solution under facility disruption, en-route congestion, and in-facility queuing. However, most of them only addressed storage facility of emergency supplies [6] or adopted covering models [7]. It is not suitable for emergency services that usually do not have fixed distribution centers. With the frequent occurrences of natural disasters and terrorist events, timeliness becomes one of the most important objectives reflecting the quality of emergency services. Using EMF to improve timeliness has been gradually concerned by many researchers, such as Araz [8], Beraldi [9], Geroliminis [10], and Ishii et al. [11]. Recent studies further constructed a nonlinear integer open location-routing model for relief distribution problems [12], put forward a typical network flow model [13], and classified these services as casualty transportation and relief distribution problems [14]. These researches provided fundament solutions to EMF planning and aroused the application of modern modeling methods in this field. In view of the accessibility to disaster areas, Salman et al. [15] adopted a tabu heuristic search algorithm for maximizing the expected demand coverage. Focusing on the problem of temporary disaster response facility allocation, Cavdur et al. [16] developed a two-stage stochastic programming to minimize the total distance traveled, the unmet demand, and the number of facilities. In addition, the transportation infrastructure and routing were also frequently considered in the EMF location problem. Typically, Chen et al. [17] applied network-based partitioning to determining temporary location for emergency medical service facility. Caunhye et al. [18] put forward a location-routing model for prepositioning and distributing emergency supplies under an uncertain demand. They enhanced the practicability and timeliness of emergency service by EMF.

Besides the location of EMF, the fleet size is also an important research topic on the emergency service. It is related closely with emergency service timeliness and service cost. Obviously, more EMFs improve service efficiency but bring more maintenance cost. To find the rational fleet size, Bell [19] developed a queuing theory model for computing the size of emergency ambulance fleet required to meet specified standards of service. Later, recognizing the complexity of the emergency service vehicle deployment, Eaton [20] found a compromise among competing objectives: minimizing response times; minimizing the number of facilities deployed; and maximizing system performance. Besides, there have also been literature works on fleet size with other factors. Özdamar [21] took into account time-dependent demand and fleet size to coordinate logistics support for relief operations. Yang [22] tried to find the proper fleet size or crew size for emergency systems when multiple objectives (minimizing cost and maximizing coverage) were considered. These existing studies are very instructive, but it was Haghani [23] who elaborated on real-time emergency response fleet deployment: concepts, systems, simulation, and case studies. Subsequently, the fleet size and location of EMF were discussed in depth. Considering that fleet size and location are the two critical factors for emergency service, Lim [24] studied the issue of EMF dispatch policies by using dynamic relocation models. Garrido [25] presented a stochastic programming model to find the fleet size that must be available at each location. These models were commonly used for deterministic emergency demand. Only in recent years did the uncertain problem of EMF fleet size and location gain attention. Moreno et al. [26] integrated facility location and fleet sizing decisions in a multiperiod, multicommodity, and multimodal context. Ünlüyurt et al. [27] confirmed that dynamic strategies dominate static ones by estimating the performance of most of the existing models via discrete event simulation. Furthermore, a related group of literature deals with such indeterminacies of service time and emergency demand [28] by stochastic, dynamic, or robust methods. Assuming that the travel time between regions follows exponential distribution with known rates, Akdogan et al. [29] proposed an approximate queuing model for location analysis of EMFs based on Markovian processes. Vladimir [30] studied the initial or "idle" site where EMFs wait for an emergency to occur. Bélanger et al. [31] proposed an optimization model in location, relocation, and dispatching of EMFs. In addition, Zhang et al. [32] explored the sustainable multidepot emergency facility location-routing problem with uncertain information.

Although there is abundant literature on EMF fleet size and location in order to minimize the response time, evacuation distance, transportation costs, uncovered demand, etc., most of them just consider the uncertainty in the demand quantity of emergency service and ignore 
many other uncertainties, such as occurrence time (the moment emergency event occurs), wait time (the time length between the occurrence of emergency event and the start of emergency service), and service time (the time length of emergency service process). Among them, reducing wait time and travel time is the key to maximum saving people's lives and property. In addition, the models seldom recognize the importance of the continuity of emergency service process; in other words, it is meaningless to interrupt the EMF service of event and replace it with another EMF. Therefore, a reasonable emergency service scheme should minimize the maintenance cost of EMFs and consider service cost, which is a two-stage programming problem. Since the maintenance cost is largely related to the fleet size of EMFs and the service cost is related to the actual service time and the actual wait time, the first stage needs to determine the fleet size of EMFs according to the multiple uncertainties of emergency service. The second stage needs to determine the appropriate EMF locations to ensure that the actual service time and the actual wait time are minimized for all emergency events.

\section{Emergency Mobile Facility Fleet Size and Location Model}

Referencing the description in reference [33], we define emergency mobile facility fleet size and location problem (EMFFSLP) in planning horizon $[0, T]$ and network $G(D, L)$, where $D=\left\{D\left(l_{i}, l_{j}\right)\right\}$ denotes a set of travel time between two locations $l_{i}, l_{j} \in L$ and $T$ is the time span or end time of all emergency services. The set $L=\{1,2, \ldots, l\}$ is all the locations where EMFs can stop. Suppose that $M=\{1,2, \ldots, m\}$ is the index set and $U$ is the resource capacity of EMFs, the model of EMFFSLP tries to minimize the total cost by finding proper fleet size and locations of EMFs under given location set $L$, distance of travel time $D$, and resource capacity $U$. The fleet size and locations of EMFs are related to the emergency events served. The higher the frequency of emergency event, the more the demand of emergency resource required. And the longer the event duration in multiple scenarios, the more the EMFs are required. Obviously, it is necessary to describe the features of emergency event in constructing the model for the EMFFSLP. Hence, we abstract $T_{e}^{O}, T_{e}^{B}, T_{e}^{D}, r_{e}$, and $l_{e}$ as the characteristics of a specific emergency event $e \in E$, where $E=\{1,2, \ldots, e\}$ is the set of all emergency events ordered by occurrence time. These characteristics are all uncertain and depend on emergency event. In this abstraction, $T_{e}^{O}$ is the occurrence time of the emergency event, $r_{e}$ is the demand quantity per unit time, and $T_{e}^{B}$ is the cutoff time when the emergency event $e$ must be served. The demand time $T_{e}^{D}$, equivalent to service time, indicates that the emergency event $e$ must be served continuously during this time, that is, service time cannot be divided into multiple time periods served with different EMFs. In other words, $T_{e}^{B}-T_{e}^{O}$ is the maximum time length that the emergency event $e$ can wait, and $r_{e} T_{e}^{D}$ is the total demand quantity of the emergency event $e$. Moreover, since any emergency event must be serviced by an EMF at a given location and each location can establish a corresponding relationship with its nearest EMF, we suppose that $l_{e} \in L$ denotes the location of the emergency event $e$.

Based on the abstraction above, considering that each EMFs must serve emergency event in turn and cannot be interrupted, we define $x_{e_{i}}^{m}\left(t_{e_{i}}\right)$ as the decision variable, where $e_{i}$ is the $i$ th emergency event served by EMF $m$ and $x_{e_{i}}^{m}\left(t_{e_{i}}\right)$ equals to 1 representing the EMF $m$ start serving the emergency event $e_{i}$ at time $t_{e_{i}}$, otherwise 0. Obviously, if collecting all $x_{e_{i}}^{m}\left(t_{e_{i}}\right)$ relative to EMF $m$ and sorting them by $i$, we can obtain the service sequence $\left\{e_{i}^{m}\right\}$ of EMF $m$. In addition, referring the model in literature [23], we suppose that the cost of emergency services includes the maintenance cost for the daily operation of all EMFs, the travel cost for the EMFs moving from $l_{e_{i}}$ to $l_{e}$, the wait cost for the actual wait time $\left(t_{e_{i}}-T_{e_{i}}^{O}\right)$, and the service cost for emergency event. Thus, we can seek reasonable fleet size and locations of emergency mobile facilities by minimizing the total cost. With all emergency services uninterrupted, the EMFFSLP is formulated as follows:

$$
\begin{aligned}
\min & f|M|+\sum_{m \in M} \sum_{e_{i} \in E} \sum_{t_{e_{i}}<T} x_{e_{i}}^{m}\left(t_{e_{i}}\right)\left[\beta D\left(l_{e_{i}} l_{e_{i-1}}\right)+\gamma\left(t_{e_{i}}-T_{e_{i}}^{O}\right)\right. \\
& \left.+\delta r_{e_{i}} T_{e_{i}}^{D}\right], \quad l_{e_{0}^{m}}=l_{m},
\end{aligned}
$$

$$
\begin{array}{ll}
\text { s.t. } \quad & \sum_{m \in M} \sum_{e_{i} \in E} \sum_{t_{e_{i}}<T} x_{e_{i}}^{m}\left(t_{e_{i}}\right) \leq|E|, \\
& \sum_{m \in M} \sum_{e_{i} \in E} \sum_{e_{e_{i}}<T} r_{e_{i}} T_{e_{i}}^{D} x_{e_{i}}^{m}\left(t_{e_{i}}\right) \leq|M| U, \\
& x_{e_{i}}^{m}\left(t_{e_{i}}\right)+x_{e_{i}}^{m^{\prime}}\left(t_{e_{i}}\right)<2, \quad m \neq m^{\prime}, \forall m \in M, \\
& x_{e_{i}}^{m}\left(t_{e_{i}}\right)+x_{e_{i+1}}^{m}\left(t_{e_{i+1}}\right)=2, \\
& \max \left(T_{e_{i+1}}^{O}, t_{e_{i}}+T_{e_{i}}^{D}+D\left(l_{e_{i}}, l_{e_{i+1}}\right)\right) \leq t_{e_{i+1}} \leq T_{e_{i+1}}^{B},
\end{array}
$$

$\forall m \in M$,

$$
\begin{aligned}
& x_{e_{1}}^{m}\left(t_{e_{1}}\right)=1, \\
& \quad \max \left(T_{e_{1}}^{O}, D\left(l_{m}, l_{e_{1}}\right)\right) \leq t_{e_{1}} \leq T_{e_{1}}^{B}, \quad \forall m \in M, \\
& x_{e_{i}}^{m}\left(t_{e_{i}}\right)+x_{e_{i+1}}^{m}\left(t_{e_{i+1}}\right) \leq 1, \\
& t_{e_{i}}+T_{e_{i}}^{D}<t_{e_{i+1}}<t_{e_{i}}+T_{e_{i}}^{D}+D\left(l_{e_{i}}, l_{e_{i+1}}\right), \quad \forall m \in M,
\end{aligned}
$$

$$
\begin{aligned}
& 0 \leq t_{e_{i}} \leq T, 0 \leq t_{e_{i+1}} \leq T, 0 \leq t_{e_{1}} \leq T, \\
& l_{e_{i}}, l_{e_{i-1}}, l_{e_{i+1}}, l_{m}, l_{e_{1}} \in L, \quad \forall m \in M .
\end{aligned}
$$

The objective function (1) aims to minimize the total cost by seeking reasonable fleet size and locations of EMFs. It indicates that $f$ is the maintenance cost in planning horizon 
$[0, T] ; \beta, \gamma$, and $\delta$ are cost coefficients, which, respectively, denote the cost per unit of travel time, the cost per unit of wait time, and the cost per unit of service time. Here, the fleet size is equal to $|M|$, the number of elements in the set $M$. And since $l_{e_{0}}$ is meaningless, it is applied to represent $l_{m}$, the initial location of EMF $m$. Constraint (2) states that the maximum numbers of emergency event will be served. Constraint (3) requires that the total demand served is less than the quantity of resources carried by all EMFs, where we suppose that each EMF has the same resource capacity. Constraint (4) stipulates that no emergency event can be served by two different EMFs. Constraint (5) guarantees the service of the $(i+1)$ th emergency event by the EMF $m$ must start after the EMF has finished the service for the $i$ th emergency event and traveled to the location $l_{e_{i+1}}$. Constraint (6) ensures that the EMF $m$ must travel from the initial location $l_{m}$ to the location $l_{e_{1}}$ before the EMF starts providing first service. Constraint (7) requires that the EMF $m$ cannot serve the emergency event $e_{i+1}$ at the location $l_{e_{i+1}}$ before time $t_{e_{i}}+T_{e_{i}}^{D}+D\left(l_{e_{i}}, l_{e_{i+1}}\right)$ because the EMF $m$ is serving $e_{i}$ or moving from $l_{e_{i}}$ to $l_{e_{i+1}}$. Here, Constraints (5) and (7) potentially guarantee that emergency services cannot be interrupted during the time $T_{e_{i}}^{D}$.

\section{Robust Optimization and Model Solution}

In the model of the EMFFSLP, $T_{e}^{O}, T_{e}^{B}, T_{e}^{D}, r_{e}$, and $l_{e}$ are not deterministic in planning horizon $[0, T]$. This kind of uncertainty is caused by many factors of emergency event with the unknown probability distribution. Since the uncertainty cannot be completely eliminated, the best way for decision makers to face the uncertainty is to accept the existence of the uncertainty problem and then analyze the problem in detail to integrate it into the decision-making model. A common method is to explore the second decision stage in the corresponding worst case from the first decision stage [34]. If the decision in the second stage is changed to a compensation decision, under the same uncertainty condition, the economic efficiency of the two-stage model will be better than that of the single-stage model [35]. However, the challenge by this method is that the model is much more difficult to solve. Therefore, as for the analysis results by Snyder [36], we adopt robust optimization to integrate the programming requirement and the uncertainty of scenarios. The objective function of the EMFFSLP thus has the following form:

$\min \{E(G)+\lambda \operatorname{Var}(G)+\omega$ (sum of squares of infeasibilities) $\}$,

where $\lambda$ and $\omega$ are risk factors and they denote the attitude of decision maker facing different risks. Thereby, the EMFFSLP in robust optimization can be presented as follows:

$$
\min f|M|+\operatorname{opt}\left(\operatorname{RP}\left(T_{e}^{O}, T_{e}^{B}, T_{e}^{D}, r_{e}, l_{e}\right)\right)
$$

Here, $\operatorname{RP}\left(T_{e}^{O}, T_{e}^{B}, T_{e}^{D}, r_{e}, l_{e}\right)$ is the optimal objective function of the compensation problem in the second stage. Let $\Omega=\{1,2, \ldots, S\}$ be the scenario set and $\varphi_{s}$ be the occurrence probability of the scenario $s \in \Omega$; we can convert the original EMFFSLP model to the formula which follows:

$$
\begin{aligned}
& \min \quad f|M|+\bar{V}+\lambda \sum_{s \in \Omega} \varphi_{s}\left(\sum_{m \in M} \sum_{e_{i} \in E^{s}} \sum_{t_{e_{i}}<T} x_{e_{i}}^{m, s}\left(t_{e_{i}}\right)\left[\beta D\left(l_{e_{i}}, l_{e_{i-1}}\right)+\gamma\left(t_{e_{i}}-T_{e_{i}}^{O}\right)+\delta r_{e_{i}} T_{e_{i}}^{D}\right]-\bar{V}\right)^{2} \\
& +\omega \sum_{s \in \Omega} \varphi_{s} \sum_{m \in M}\left(U-\sum_{e_{i} \in E^{s}} \sum_{t_{e_{i}}<T} r_{e_{i}} T_{e_{i}}^{D} x_{e_{i}}^{m, s}\left(t_{e_{i}}\right)\right)^{2}, \\
& \bar{V}=\sum_{s \in \Omega} \varphi_{s} \sum_{m \in M} \sum_{e_{i} \in E^{s}} \sum_{t_{e_{i}}<T} x_{e_{i}}^{m, s}\left(t_{e_{i}}\right)\left[\beta D\left(l_{e_{i}}, l_{e_{i-1}}\right)+\gamma\left(t_{e_{i}}-T_{e_{i}}^{O}\right)+\delta r_{e_{i}} T_{e_{i}}^{D}\right], \quad l_{e_{0}^{m}}=l_{m}, \\
& \text { s.t. } \quad \sum_{m \in M} \sum_{e_{i} \in E^{s}} \sum_{t_{e_{i}}<T} x_{e_{i}}^{m, s}\left(t_{e_{i}}\right) \leq\left|E^{s}\right|, \quad \forall s \in \Omega, \\
& \sum_{m \in M} \sum_{e_{i} \in E^{s}} \sum_{t_{e_{i}}<T} r_{e_{i}} T_{e_{i}}^{D} x_{e_{i}}^{m, s}\left(t_{e_{i}}\right) \leq|M| U, \quad \forall s \in \Omega,
\end{aligned}
$$

(4), (5), (6), (7), (8), $e_{i} \in E^{\mathcal{s}}, \forall s \in \Omega, \forall m \in M$.

Since we try to satisfy all demands of the emergency events in scenarios, the value of $\sum_{s \in \Omega} \varphi_{s} \sum_{m \in M}\left(U-\sum_{e_{i} \in E^{s}} \sum_{t_{e_{i}}<T} r_{e_{i}}\right.$ $\left.T_{e_{i}}^{D} x_{e_{i}}^{m, s}\left(t_{e_{i}}\right)\right)^{2}$ is a fixed value with the given scenario set $\Omega$. It does not affect the determination of the fleet size and locations in terms of the total cost minimized, so it can be deleted from formula (11). Hence, we reduce formula (11) to formula (15): 


$$
\min f|M|+\bar{V}+\lambda \sum_{s \in \Omega} \varphi_{s}\left(\sum_{m \in M} \sum_{e_{i} \in E^{s}} \sum_{t_{e_{i}}<T} x_{e_{i}}^{m, s}\left(t_{e_{i}}\right)\left[\beta D\left(l_{e_{i}}, l_{e_{i-1}}\right)+\gamma\left(t_{e_{i}}-T_{e_{i}}^{O}\right)+\delta r_{e_{i}} T_{e_{i}}^{D}\right]-\bar{V}\right)^{2} .
$$

Obviously, the introduction of the expectation and variance in formula (15) causes much computational complexity. It is difficult for software packages such as LINDO to solve, while swarm intelligence algorithms are hard to be applied directly in this kind of global cost minimization problem based on the second-stage minimization. Therefore, we modify ant colony algorithm by ant teams to decompose the robust optimization model. The inputs of the ant colony algorithm are travel time matrix $D\left(l_{i}, l_{j}\right)$, the EMF set $M$, the resource capacity $U$, the scenario set $\Omega$, the occurrence possibility of scenario $\varphi_{s}$ and the emergency event characteristics $T_{e}^{O}, T_{e}^{B}, T_{e}^{D}$, $r_{e}$, and $l_{e}$. Let $\lambda(\beta+\gamma+\delta)$ denote the risk reorganization factor, and we put forward an ant colony algorithm in 5 steps to solve the robust optimization model of the EMFFSLP.

4.1. Step 1 (Ant Team Coding). We divide ants in the algorithm into $k_{\max }-k_{\min }+1$ teams, where $k_{\min }=\min _{s \in \Omega}$ $\left(\sum_{e_{i} \in E^{s}} r_{e_{i}} T_{e_{i}}^{D}\right) / U$ and $k_{\max }=E^{M}, E^{M}=\max \left(\left|E^{s}\right|\right)$. Each team has $k\left|L \| E^{M}\right|$ ants and represents the solution in a certain number of EMFs $|M|$. The $h$ th ant in the team $k$ is marked as a string $A_{h, k}, k \in\left\{k_{\min }, k_{\min }+1, \ldots, k_{\max }\right\}$, where $h \in$ $\left\{1,2, \ldots, k|L|\left|E^{M}\right|\right\}$. Each ant has $k$ sections of service information which construct the solution when the number of EMFs $|M|$ is $k$. Each section $l_{m}, e_{1}^{m}, t_{1}^{m}, \ldots, e_{i}^{m}, t_{i}^{m}$ denotes that the EMF $m$ starts serving the $i$ th emergency event $e_{i}^{m}$ at time $t_{i}^{m}$ and the initial location $l_{m}$.

Thus, the complete information of an ant is $l_{1}, e_{1}^{1}$, $t_{1}^{1}, \ldots, e_{i}^{1}, t_{i}^{1}, l_{2}, e_{1}^{2}, t_{1}^{2}, \ldots, e_{j}^{1}, t_{j}^{1}, l_{k}, e_{1}^{k}, t_{1}^{k}, \ldots e_{z}^{1}, t_{z}^{1}$, which is also a solution at $|M|=k$. Here, we use $e_{i}^{m}, t_{i}^{m}$ instead of $x_{e_{i}}^{m}\left(t_{e_{i}}\right)$ to simplify the description of the section of ant, where $e_{i}^{m}$ is the $i$ th emergency event served by the EMF $m, l_{m}$ is the initial location of EMF $m$, and $t_{i}^{m}$ is the service start time of $e_{i}^{m}$. If $x_{e_{i}}^{m}\left(t_{e_{i}}\right)=1$, the corresponding section of the ant will be recorded; otherwise it will be deleted.

4.2. Step 2 (Ant Initialization). For the $h$ th ant in the ant team $k$, we define an empty ant string (solution) of $A_{h, k}=$ null. Let $\mu \in \mathrm{R}^{+}$is a random positive integer with uniform distribution, we copy the set $\cup_{s} E^{s}$ to $E_{h, k}$ as the emergency events that need to be served and assign the location $l_{\mu \%|L|+1}$ as the initial location $l_{i}$ of the $i$ th section of the $h$ th ant, so that $k|L|\left|E^{M}\right|$ ants are equally allocated to different locations. These locations are appended into the corresponding sections of the solution $A_{h, k}$; thus, $A_{h, k}=$ $l_{1}, \ldots, l_{k}$. Then, we randomly choose an emergency event from $E_{h, k}$ as the first emergency event $e_{1}^{1}$ to be served. Since the solution $A_{h, k}$ is empty except the initial locations, the emergency event $e_{1}^{1}$ definitely meets all constraints while
$T_{e_{1}}^{B} \geq \max \left(D\left(l_{1}, l_{e_{1}}\right), T_{e_{1}}^{O}\right)$. At this point, we delete the emergency event $e_{1}^{1}$ from the set $E_{h, k}$ and append this service information into the solution, and otherwise, we try to choose another emergency event from $E_{h, k}$. Thus, the solution $A_{h, k}$ is updated to $l_{1}, e_{1}^{1}, t_{1}^{1}, l_{2}, \ldots, l_{k}$, where $t_{1}^{1}=$ $\min \left(\max \left(D\left(l_{1}, l_{e_{1}}\right), T_{e_{1}}^{O}\right), T_{e_{1}}^{B}\right)$. If we cannot find an emergency event $e_{1}^{1}$ under $T_{e_{1}}^{B} \geq \max \left(D\left(l_{1}, l_{e_{1}}\right), T_{e_{1}}^{O}\right)$, this means the ant $A_{h, k}$ is invalid, so it will be deleted from the ant team.

4.3. Step 3 (Pheromone Update). Considering that EMFs have an equal opportunity to provide service, we set initial pheromone $\tau_{e_{i}, e_{j}}$ to $1 / C_{\left|U_{s} E^{s}\right|}^{2}$ in order to uniformly distribute the pheromones on each path. The paths here are the time distance between locations. With the optimization by ant foraging, the pheromones on these paths increase gradually, so it is suitable for adopting an online update method to modify the pheromones according to the emergency service. Thus, we define the transit function of pheromones as $\tau_{e_{j}, e_{j+1}}(t)=(1-\rho) \tau_{e_{j}, e_{j+1}}+\sum_{h} \sum_{k} \Delta \tau_{e_{j}, e_{j+1}}^{h, k}$, where $0<\rho \leq 1$ is the volatility of pheromone and $\Delta \tau_{e_{j}, e_{j+1}}^{h, k}$ is the pheromone left on the path from the location $l_{e_{j}}$ to $l_{e_{j+1}}$ when ant $A_{h, k}$ has served the emergency events $e_{j}$ and $e_{j+1}$. The value of $\Delta \tau_{e_{j}, e_{j+1}}^{h, k}$ is $\left(D\left(l_{e_{j}}, l_{e_{j+1}}\right)+T_{e_{j}}^{D}\right)^{-1}$ representing the inverse of the time distance from $l_{e_{j}}$ to $l_{e_{j+1}}$ plus the demand time of $e_{j}$. Here, we do not distinguish the difference in the number of EMFs when we sum up the pheromones of all ants. It ensures that any ant in one team can be optimized by referencing the partial path of other ant teams.

4.4. Step 4 (Selection Probability). For any EMF $m$, it corresponds to the $m$ th section of ant; let $a$ and $b$ be the weight of pheromone and visibility. We set the service transition probability from emergency event $e_{j}$ to emergency event $e_{j+1}$ as $P_{e_{j}, e_{j+1}}^{\theta}(t)=\left[\tau_{e_{j} e_{j+1}}(t)\right]^{a} \times\left[\eta_{e_{j}, e_{j+1}}(t)\right]^{b} / \sum_{e_{i} \in E_{h, k},}\left[\tau_{e_{j}, e_{i}}(t)\right]^{a} \times$ $\left[\eta_{e_{j}, e_{i}}(t)\right]^{b}$, where $\theta$ is the number of iterations and $\eta_{e_{j}, e_{j}}(t)=$ $1 / D\left(l_{e_{j}}, l_{e_{j+1}}\right)$ and $\eta_{e_{j}, e_{i}}(t)=1 / D\left(l_{e_{j}}, l_{e_{i}}\right)$ are ant visibility (we define $\eta_{e_{i}, e_{i}}(t)=1$ when $\left.D\left(l_{e_{j}}, l_{e_{i}}\right)=0\right)$. After the emergency event $e_{j}$ is served, we choose an emergency event $e_{i}$ in $E_{h, k}$ according to the descending order of $\left\{P_{e_{j}, e_{i+1}}^{\theta}(t)\right\}$ and create a random probability $P_{i}$. If $P_{i}$ is in the interval $\left(\sum_{\psi<i} P_{e_{i}, e_{\psi}}^{\theta}(t), \sum_{\psi \leq i} P_{e_{i}, e_{\psi}}^{\theta}(t)\right)$ and we can find $e_{i}$ and $t \in[0, T]$ meets the constraints (4) (8) and (13) with time step $\xi$, the emergency event $e_{i}$ is marked as the next emergency event $e_{j+1}^{m}$ and $t$ is the start time $t_{j+1}^{m}$ of the emergency service. At this point, we delete $e_{j+1}^{m}$ from $E_{h, k}$ and modify solution $A_{h, k}$ by appending the service information of $e_{j+1}^{m}$. If the pairs of $e_{i}$ and $P_{i}$ cannot satisfy the constraints 
above, we try to create another pairs till all emergency events in $E_{h, k}$ are checked. In case if no suitable pairs are found, the solution $A_{h, k}$ will be considered as unreasonable and be deleted from ant teams. Here, we do not need check whether $e_{i}$ satisfies the constraints (12); it is due to the fact that ant teams are constructed according to $k_{\max }$ and $k_{\min }$, and the ant team coding has already guaranteed that at most all emergency events in $E_{h, k}$ will be served.

4.5. Step 5 (Terminal Condition). The algorithm terminates when there is no emergency event in all copies $E_{h, k}$ from the set $\cup_{s} E^{s}$ that can be served. At this time, we sort all ants according to formula (15). The ant with the minimum cost will be the preferred solution of the EMFFSLP.

\section{Analysis of Examples}

In order to verify the practice of the model, we set up 12 locations in Xianyang city, China, where EMFs can stop, as shown in Figure 1. The minimum time distance between two locations is $5 \mathrm{~min}$, the maximum is $30 \mathrm{~min}$, and the average is $15 \mathrm{~min}$. Let the plan horizon $T=24$ hours; we suppose that the maintenance cost of an EMF (including equipment cost, insurance costs, repair cost, and manpower cost) is $¥ 181000$ per year; that is to say, $f=495$. In the case of the earthquake event, we split the emergency events under 24 scenarios. Each scenario has different numbers of emergency events. The maximum number of emergency events in scenarios $\max (|E|)$ is 38 . The occurrence probability of scenarios, as shown in Table 1, is in the interval $[0.002,0.076]$. The longest wait time of emergency events is less than 4 hours, and the greatest demand quantity is less than 300 units. The detail information of Scenario 1 is shown in Table 2 and Figure 1. The demand time of emergency events in Scenario 1 is between $10 \mathrm{~min}$ and $50 \mathrm{~min}$, and most emergency events are close to the locations $l_{4}, l_{5}, l_{7}$, and $l_{9}$.

Given the resource capacity of each EMF $U=1300$ units and the risk reorganization factor $\lambda(\beta+\gamma+\delta)=0.76$, we obtain $k_{\min }=2$ and $k_{\max }=38$ which constructs the interval of the number of EMFs. With the volatility of pheromone $\rho=0.5$ and the parameters $a=1$ and $b=2$, we generate 37 ant teams in the ant colony algorithm. Each ant team has $456 \times k$ ants and $k \in[2,38]$. After $\theta=5296158$ iterations, we obtain a preferred solution to the EMFFSLP under the time step $\xi=1 \mathrm{~min}$. As shown in Figure 1, we can see that

(1) The 6 EMFs initially stop at locations $l_{2}, l_{3}, l_{5}, l_{6}, l_{7}$, and $l_{10}$ marked green. These initial locations, covering $66.7 \%$ emergency events, are in the regions where emergency events frequently happen. For example, the number of emergency events belonging to location $l_{3}$ and $l_{7}$ is up to 5 . The location $l_{4}$ is the closest to the initial location of EMFs $\left\{l_{2}, l_{3}, l_{5}, l_{6}, l_{7}, l_{10}\right\}$, and the time distance is only 5 mins. Even though the location $l_{9}$ is the farthest to the initial location of EMFs, the time distance is 8 mins. According to the computational results of locations, it is shown that the $6 \mathrm{EMFs}$ are scattered in the whole Xianyang city, and that they can reach any location of emergency events in a short time. It is convenient to quickly provide emergency service in the city.

(2) Taking the locations of the 6 EMFs as centers, we draw 6 control ranges labeling the scopes that EMFs can reach in $11 \mathrm{~min}$ (the most common time distance between two initial locations of EMFs). The control ranges cover $100 \%$ area of Xianyang city. That is to say, the 6 EMFs assigned at specific locations can timely handle all emergency events, even though the location set $L$ is changed. Specially, in the regions with dense population and high earthquake risk, there are 12 emergency events $e_{2}, e_{3}, e_{7}, e_{15}, e_{16}$, $e_{18}, e_{23}, e_{25}, e_{27}, e_{33}, e_{35}$, and $e_{36}$ covered by 3 EMFs. It indicates that multi-EMFs can collaboratively deal with these emergency events, further reducing the service time and promoting the service ability.

(3) As shown in Figure 2, if the preferred solution is applied to Scenario 1, we can see that all demands of the emergency events are satisfied. The 6 EMFs $m_{1} \sim m_{6}$ respectively handle 7,6,6,5,7, and 5 emergency events. The total travel time is 185 mins, and the total actual wait time is only 448 mins which saves 1157 mins from total emergency services. It demonstrates that the solution makes the most of the uncertainty characteristics of emergency events. If we choose the suboptimal solution from the results, namely, the solution with 7 EMFs by adding an EMF at location $l_{4}$, the EMFs will cover more emergency events. At this point, the maximum time distance of EMFs remains unchanged, but the average time distance decreases to 14 mins. The introduction of new EMF and the reduction of time distance save the service time cost. However, the maintenance cost of an EMF is much greater than the benefit of reducing service time. Similarly, if we choose the solution with 5 EMFs by reducing the EMF at location $l_{10}$, the service ability of EMFs is certainly declined. Although the maintenance cost in EMFs is reduced, the emergency events at location $l_{10}$ and $l_{8}$ are far away from the locations of EMFs. It takes $14 \mathrm{~min}$ for the nearest EMF to move to location $l_{8}$ and $12 \mathrm{~min}$ to move to location $l_{10}$. In other words, the fewer EMFs bring much more service cost in emergency service. Hence, the solution with 6 EMFs is the optimal solution to the EMFFSLP.

(4) Generally speaking, it is unreasonable to distribute the EMFs on the boundary (the locations on the convex hull of the research area), because the mobility on the boundary is relatively less than that inside the research area. However, when the location on the boundary is closer to other locations and emergency events, the best way is to assign some EMFs on the boundary so as to handle multiple emergency events. As shown in Figure 1, the preferred solution with 6 EMFs chooses more suitable locations for EMFs according to the distribution of 


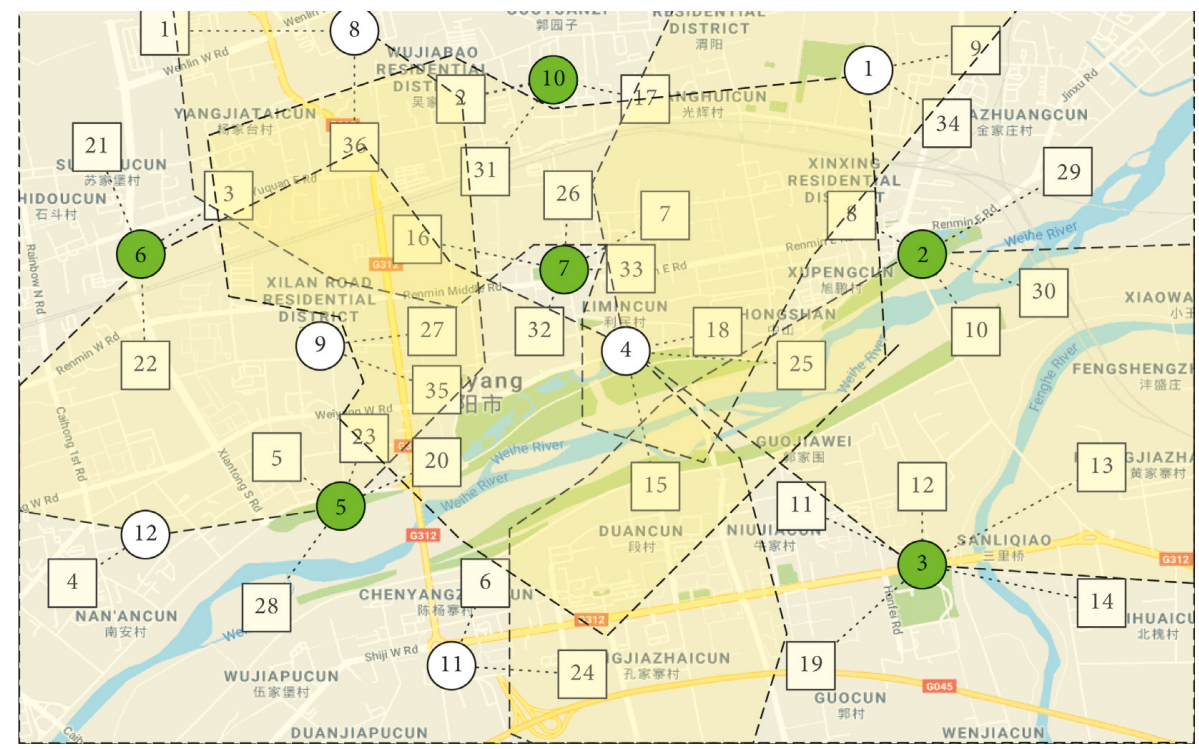

$D(1,2)=8 \mathrm{~min} ; D(1,3)=14 \mathrm{~min} ; D(1,4)=13 \mathrm{~min} ; D(1,5)=21 \mathrm{~min} ; D(1,6)=25 \mathrm{~min} ; D(1,7)=11 \mathrm{~min} ; D(1,8)=17 \mathrm{~min}$; $D(1,9)=20 \mathrm{~min} ; D(1,10)=11 \mathrm{~min} ; D(1,11)=22 \mathrm{~min} ; D(1,12)=25 \mathrm{~min} ; D(2,3)=11 \mathrm{~min} ; D(2,4)=11 \mathrm{~min} ; D(2,5)=19 \mathrm{~min}$; $D(2,6)=19 \mathrm{~min} ; D(2,7)=12 \mathrm{~min} ; D(2,8)=23 \mathrm{~min} ; D(2,9)=20 \mathrm{~min} ; D(2,10)=15 \mathrm{~min} ; D(2,11)=17 \mathrm{~min} ; D(2,12)=22 \mathrm{~min}$; $D(3,4)=15 \mathrm{~min} ; D(3,5)=14 \mathrm{~min} ; D(3,6)=22 \mathrm{~min} ; D(3,7)=19 \mathrm{~min} ; D(3,8)=30 \mathrm{~min} ; D(3,9)=19 \mathrm{~min} ; D(3,10)=23 \mathrm{~min}$; $D(3,11)=12 \mathrm{~min} ; D(3,12)=16 \mathrm{~min} ; D(4,5)=11 \mathrm{~min} ; D(4,6)=15 \mathrm{~min} ; D(4,7)=5 \mathrm{~min} ; D(4,8)=20 \mathrm{~min} ; D(4,9)=11 \mathrm{~min}$; $D(4,10)=9 \min ; D(4,11)=12 \min ; D(4,12)=13 \min ; D(5,6)=11 \min ; D(5,7)=13 \min ; D(5,8)=20 \min ; D(5,9)=8 \mathrm{~min} ;$ $D(5,10)=20 \mathrm{~min} ; D(5,11)=7 \mathrm{~min} ; D(5,12)=5 \mathrm{~min} ; D(6,7)=15 \mathrm{~min} ; D(6,8)=11 \mathrm{~min} ; D(6,9)=8 \mathrm{~min} ; D(6,10)=14 \mathrm{~min}$ $D(6,11)=16 \mathrm{~min} ; D(6,12)=11 \mathrm{~min} ; D(7,8)=14 \mathrm{~min} ; D(7,9)=12 \mathrm{~min} ; D(7,10)=12 \mathrm{~min} ; D(7,11)=14 \mathrm{~min}$; $D(7,12)=15 \mathrm{~min} ; D(8,9)=16 \mathrm{~min} ; D(8,10)=6 \mathrm{~min} ; D(8,11)=22 \mathrm{~min} ; D(8,12)=22 \mathrm{~min} ; D(9,10)=15 \mathrm{~min}$; $D(9,11)=10 \min ; D(9,12)=8 \min ; D(10,11)=20 \min ; D(10,12)=21 \mathrm{~min} ; D(11,12)=11 \mathrm{~min}$

(i): Location

$j$ : Emergency event $-\cdots$
$\cdots \quad:$ : Affiliation
$\vdots$ Control range

FIGURE 1: Solution to the EMFFSLP.

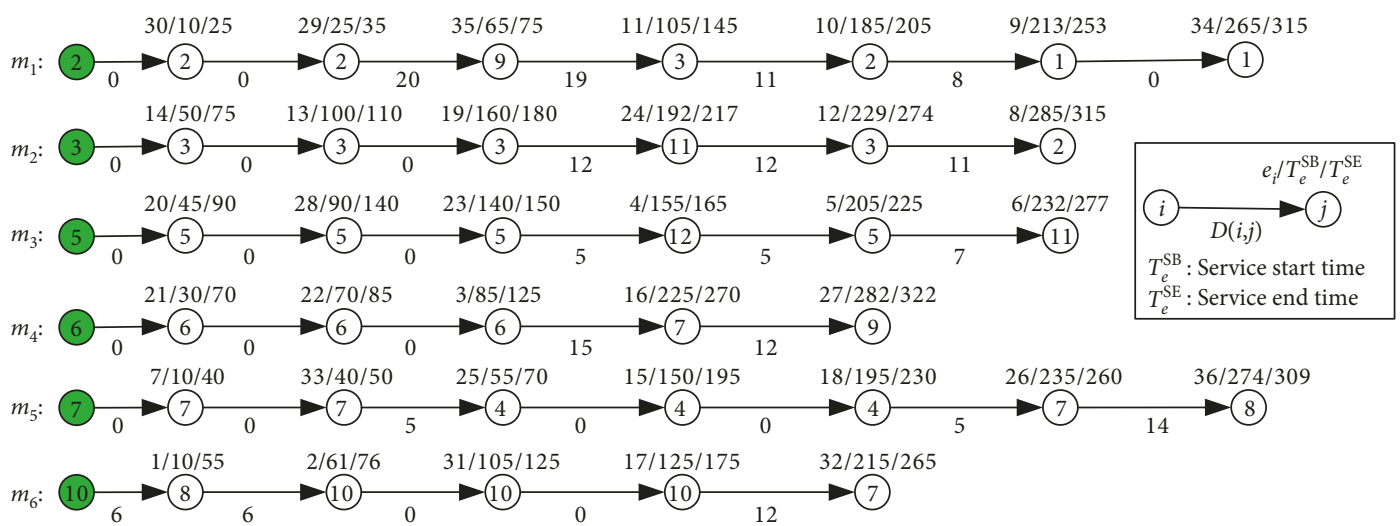

Figure 2: Routing of the 6 EMFs in Scenario 1.

the 12 locations and the 24 scenarios of emergency events. For example, the selected locations $l_{2}, l_{3}$, and $l_{6}$ for the EMFs are on the boundary, and the selected locations $l_{5}, l_{7}$, and $l_{10}$ are inside the research area. It helps EMFs quickly travel to any location.

(5) The location of EMFs depends not only on the number of EMFs but also on the number of locations. Particularly, the location containing more emergency events is more likely to be an initial location of EMF. As the Scenario 1 shown in Figure 1, the EMF at location $l_{4}$ can deal with the emergency events $e_{15}, e_{18}, e_{25}$, and $e_{38}$ or the emergency events $e_{7}, e_{16}, e_{26}, e_{32}$, and $e_{33}$ by moving to location $l_{7}$. The EMF at location $l_{7}$ can deal with the emergency events $e_{7}, e_{16}, e_{26}, e_{32}$, and $e_{33}$ and the emergency events $e_{2}, e_{17}$, and $e_{31}$ by moving to location $l_{10}$ or the emergency events $e_{15}, e_{18}, e_{25}$, and $e_{38}$ by moving to location $l_{4}$. Therefore, the solution has an EMF at location $l_{7}$ 


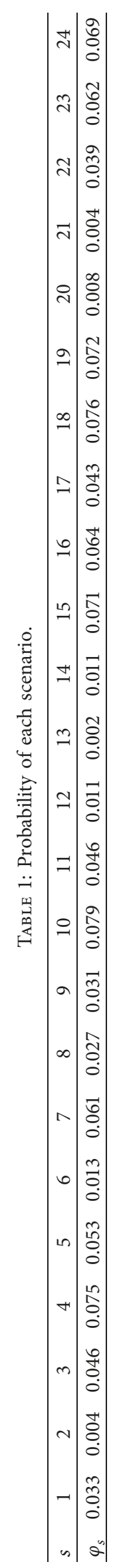


TABLE 2: Information on all emergency events in Scenario 1.

\begin{tabular}{|c|c|c|c|c|c|c|c|c|c|c|c|c|c|c|c|c|c|}
\hline$e$ & $T_{e}^{O}$ & $T_{e}^{B}$ & $T_{e}^{D}$ & $r_{e}$ & $l_{e}$ & $e$ & $T_{e}^{O}$ & $T_{e}^{B}$ & $T_{e}^{D}$ & $r_{e}$ & $l_{e}$ & $e$ & $T_{e}^{O}$ & $T_{e}^{B}$ & $T_{e}^{D}$ & $r_{e}$ & $l_{e}$ \\
\hline 1 & 10 & 50 & 45 & 3 & 8 & 13 & 100 & 310 & 10 & 1 & 3 & 25 & 30 & 60 & 15 & 2 & 4 \\
\hline 2 & 15 & 90 & 15 & 2 & 10 & 14 & 50 & 110 & 25 & 2 & 3 & 26 & 235 & 260 & 25 & 3 & 7 \\
\hline 3 & 70 & 150 & 40 & 3 & 6 & 15 & 150 & 175 & 45 & 4 & 4 & 27 & 260 & 285 & 40 & 1 & 9 \\
\hline 4 & 135 & 175 & 10 & 1 & 12 & 16 & 150 & 245 & 45 & 2 & 7 & 28 & 80 & 95 & 50 & 5 & 5 \\
\hline 5 & 205 & 220 & 20 & 2 & 5 & 17 & 115 & 135 & 50 & 1 & 10 & 29 & 20 & 120 & 10 & 1 & 2 \\
\hline 6 & 215 & 245 & 45 & 4 & 11 & 18 & 180 & 200 & 35 & 3 & 4 & 30 & 10 & 30 & 15 & 1 & 2 \\
\hline 7 & 10 & 30 & 30 & 2 & 7 & 19 & 160 & 190 & 20 & 2 & 3 & 31 & 105 & 150 & 20 & 2 & 10 \\
\hline 8 & 245 & 285 & 30 & 2 & 2 & 20 & 45 & 70 & 45 & 2 & 5 & 32 & 215 & 235 & 50 & 1 & 7 \\
\hline 9 & 190 & 280 & 40 & 3 & 1 & 21 & 30 & 90 & 40 & 3 & 6 & 33 & 20 & 50 & 10 & 1 & 7 \\
\hline 10 & 185 & 205 & 20 & 1 & 2 & 22 & 35 & 75 & 15 & 1 & 6 & 34 & 265 & 285 & 50 & 6 & 1 \\
\hline 11 & 105 & 130 & 40 & 2 & 3 & 23 & 85 & 165 & 10 & 1 & 5 & 35 & 65 & 105 & 10 & 2 & 9 \\
\hline 12 & 195 & 260 & 45 & 3 & 3 & 24 & 160 & 230 & 25 & 2 & 11 & 36 & 245 & 280 & 35 & 2 & 8 \\
\hline
\end{tabular}

other than $l_{4}$ since it can deal with 3 more emergency events.

(6) In order to analyze the time consumption of the modified ant colony algorithm, we conducted experiments under different scenarios and in the locations where EMFs can stop. As shown in Table 3, the time consumption increases with the number of scenarios and the number of locations. It took about 3 to 15 minutes to calculate the results. Doubling the number of scenarios increased the time consumption by about $30 \%$. However, doubling the number of the locations increased the time consumption by about $90 \%$. This is due mainly to the locations bearing a significant impact on the search scope of the solution and the complexity of the pheromone update.

\section{Analysis of Risk Recognition Factor}

The illustrative example demonstrates the strategy plan of EMFs with emergency events uncertainty. However, in the practical process, the decision maker's attitude to risk also impacts the programming model. In formula (15), $\lambda$ is the risk factor affecting the fleet size and locations of EMFs. It represents the extent to which decision-makers view risk. In order to simplify analysis, we integrate the risk factor $\lambda$ and the cost coefficients into risk recognition factor (RRF) $\varepsilon$, where $\varepsilon=\lambda(\beta+\gamma+\delta)$. It indicates the cognition of time cost, construction risk, and weight value. Then, we explore the $\operatorname{RRF} \varepsilon$ so as to provide decision-makers with a more reasonable solution with various risk control parameters.

6.1. Emergency Mobile Facility Fleet Size. Aiming at analyzing the relationship between RRF and the fleet size of EMFs, we select 18 RRFs for analysis. As shown in Figure 3(a), the fleet size increases step by step with the RRF and can be sustained under a certain range. This suggests that the increase of RRF causes decision-makers providing more EMFs to avoid the risk in scenarios. But, the number of EMFs cannot increase endlessly, owing to that too much EMFs will lead to more maintenance cost produced by higher RRF. In other words, the fleet size has some tolerability to RRF. The larger the RRF, the more the tolerability of the fleet size. Let $Q(|M|)$ represent the change degree of
TABLE 3: Time consumption of the ant colony algorithm under different locations and scenarios.

\begin{tabular}{lcccc}
\hline Locations & Scenarios & Fleet size & Iterations & $\begin{array}{c}\text { Time consumed } \\
\text { (second) }\end{array}$ \\
\hline 12 & 24 & 6 & 5296158 & 189 \\
12 & 48 & 6 & 8435632 & 236 \\
12 & 96 & 7 & 10250988 & 322 \\
24 & 24 & 9 & 13534531 & 362 \\
24 & 48 & 9 & 19234455 & 510 \\
24 & 96 & 11 & 25243509 & 791 \\
\hline
\end{tabular}

the fact that RRF can be tolerated by $|M|$ EMFs; we can see that $Q(|M|)$ is positively correlated with $|M|$. This suggests that the cost of large fleet size of EMFs depends mainly on its maintenance cost other than RRF and that RRF can be ignored under unlimited capital support.

6.2. Emergency Mobile Facility Location. Under higher RRF, a small change in service time will lead to a much more impact on total cost. Thus, a feasible method is to assign the locations of EMFs as reasonable as possible, which can counteract the cost arising from service time. As shown in Figure 3(b), the minimum distance between two locations representing the dispersion of EMFs gradually increases with RRF. However, constrained by the time distance matrix $D$, EMFs cannot be scattered endlessly. Therefore, when the current EMFs is scattered enough, there are two processes to handle with the further increase of RRF. One process is to introduce a new EMF and ensure that the maintenance cost of the new EMF is less than the decreased additional cost by relocating EMFs. In this case, as shown in the $\varepsilon_{1}$ section of the curve, the minimum distance mutates to a smaller value and then gradually grows up with RRF. The other process takes place when the maintenance cost of a new EMF is more than the decreased additional cost by relocating EMFs. In this case, the minimum distance between the locations is almost unchanged, as shown in the $\varepsilon_{2}$ section of the curve.

\section{Conclusion}

Emergency services are quick responses to emergency events. It often requires decision makers to distribute an amount of EMFs to maximally save lives and properties. 


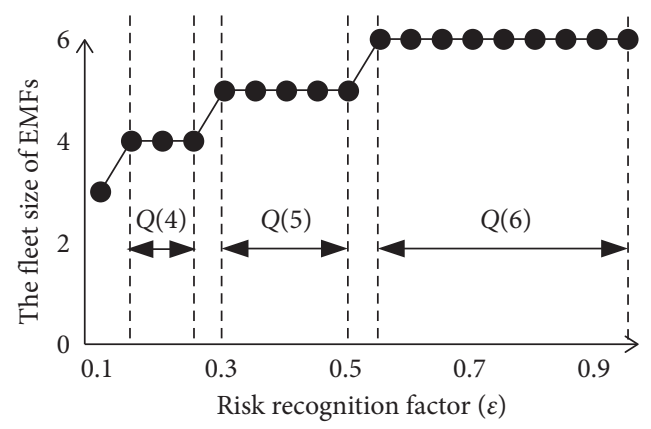

(a)

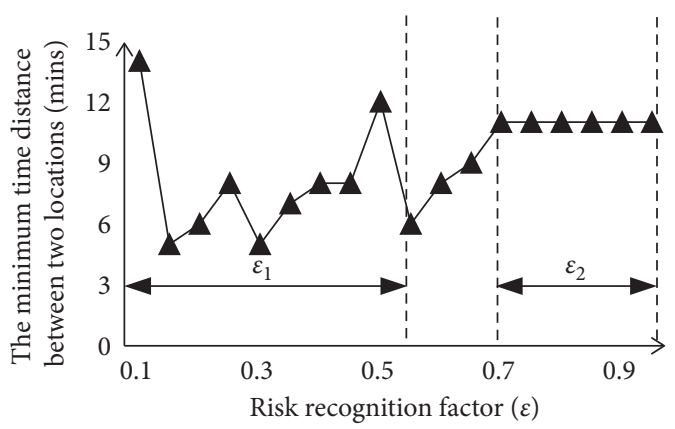

(b)

FIGURE 3: Fleet size and locations of EMFs with different RRFs. (a) Fleet size of EMFs with different RRFs. (b) Locations of EMFs with different RRFs.

Considering the uncertainty of emergency event and the noninterruptible requirements of emergency service, we propose a two-stage programming model for the EMFFSLP. The objective is to minimize the service time cost and the maintenance cost of EMFs by exploring a proper fleet size and locations of EMFs. Based on robust optimization, a modified ant colony algorithm was presented to solve the EMFFSLP and analyze the control strategy of the RRF. It presents a preferred solution to the determination of the fleet size and locations of EMFs. Since various resource capacities greatly increase the constraint complexity of the EMFFSLP model, it is difficult to obtain a satisfactory solution by using ant colony algorithm. Hence, the next step is to discuss the EMFFSLP under nonuniform resource capacities and to solve the problem with high-performance computing.

\section{Data Availability}

The program code and data that support the plots discussed within this paper are available from the corresponding author upon request.

\section{Conflicts of Interest}

The authors declare that they have no conflicts of interest.

\section{Acknowledgments}

Jianxun Li gratefully acknowledges the support of the Chinese Postdoctoral Science Fund (no. 2014M562441) and the Youth Social Science Academic Innovation Team Fund of Xi'an University of Technology: Circular Economy and Smart Regional Development under the Integration of New Technology (no. 105-451215007).

\section{References}

[1] C. Toregas, R. Swain, C. ReVelle, and L. Bergman, "The location of emergency service facilities," Operations Research, vol. 19, no. 6, pp. 1363-1373, 1971.

[2] C. G. Rawls and M. A. Turnquist, "Pre-positioning of emergency supplies for disaster response," Transportation Research Part B: Methodological, vol. 44, no. 4, pp. 521-534, 2010.
[3] C. ReVelle, D. Bigman, D. Schilling et al., "Facility location: a review of context-free and EMS models," Health Services Research, vol. 12, no. 2, p. 129, 1977.

[4] A. Döyen, N. Aras, and G. Barbarosoğlu, "A two-echelon stochastic facility location model for humanitarian relief logistics," Optimization Letters, vol. 6, no. 6, pp. 1123-1145, 2012.

[5] S. An, N. Cui, Y. Bai, W. Xie, M. Chen, and Y. Ouyang, "Reliable emergency service facility location under facility disruption, en-route congestion and in-facility queuing," Transportation Research Part E: Logistics and Transportation Review, vol. 82, no. 8, pp. 199-216, 2015.

[6] B. Balcik and B. M. Beamon, "Facility location in humanitarian relief," International Journal of Logistics Research and Applications, vol. 11, no. 2, pp. 101-121, 2008.

[7] X. Li, Z. Zhao, X. Zhu, and T. Wyatt, "Covering models and optimization techniques for emergency response facility location and planning: a review," Mathematical Methods of Operations Research, vol. 74, no. 3, pp. 281-310, 2011.

[8] C. Araz, H. Selim, and I. Ozkarahan, "A fuzzy multi-objective covering-based vehicle location model for emergency services," Computers \& Operations Research, vol. 34, no. 3, pp. 705-726, 2007.

[9] P. Beraldi and M. E. Bruni, "A probabilistic model applied to emergency service vehicle location," European Journal of Operational Research, vol. 196, no. 1, pp. 323-331, 2009.

[10] N. Geroliminis, K. Kepaptsoglou, and M. G. Karlaftis, “A hybrid hypercube genetic algorithm approach for deploying many emergency response mobile units in an urban network," European Journal of Operational Research, vol. 210, no. 9, pp. 287-300, 2011.

[11] H. Ishii and Y. L. Lee, "Mathematical ranking method for emergency facility location problem with block-wisely different accident occurrence probabilities," Procedia Computer Science, vol. 22, pp. 1065-1072, 2013.

[12] H. Wang, L. Du, and S. Ma, "Multi-objective open locationrouting model with split delivery for optimized relief distribution in post-earthquake," Transportation Research Part E: Logistics and Transportation Review, vol. 69, no. 6, pp. 160179, 2014.

[13] D. Khayal, R. Pradhananga, S. Pokharel, and F. Mutlu, "A model for planning locations of temporary distribution facilities for emergency response," Socio-Economic Planning Sciences, vol. 52, no. 9, pp. 22-30, 2015.

[14] M. Safeer, S. P. Anbuudayasankar, K. Balkumar, and K. Ganesh, "Analyzing transportation and distribution in 
emergency humanitarian logistics," Procedia Engineering, vol. 97, no. 12, pp. 2248-2258, 2014.

[15] F. S. Salman and E. Yücel, "Emergency facility location under random network damage: insights from the Istanbul case," Computers \& Operations Research, vol. 62, no. 9, pp. 266-281, 2015.

[16] F. Cavdur, M. Kose-Kucuk, and A. Sebatli, "Allocation of temporary disaster response facilities under demand uncertainty: an earthquake case study," International Journal of Disaster Risk Reduction, vol. 19, no. 8, pp. 159-166, 2016.

[17] A. Y. Chen and T.-Y. Yu, "Network based temporary facility location for the emergency medical services considering the disaster induced demand and the transportation infrastructure in disaster response," Transportation Research Part B: Methodological, vol. 91, no. 6, pp. 408-423, 2016.

[18] A. M. Caunhye, Y. Zhang, M. Li, and X. Nie, "A locationrouting model for prepositioning and distributing emergency supplies," Transportation Research Part E: Logistics and Transportation Review, vol. 90, no. 11, pp. 161-176, 2016.

[19] C. E. Bell and D. Allen, "Optimal planning of an emergency ambulance service,” Socio-Economic Planning Sciences, vol. 3, no. 2, pp. 95-101, 1969.

[20] D. J. Eaton, M. S. Daskin, D. Simmons, B. Bulloch, and G. Jansma, "Determining emergency medical service vehicle deployment in Austin, Texas," Interfaces, vol. 15, no. 1, pp. 96-108, 1985.

[21] L. Özdamar, E. Ekinci, and B. Küçükyazici, "Emergency logistics planning in natural disasters," Annals of Operations Research, vol. 129, no. 1-4, pp. 217-245, 2004.

[22] S. Yang, Integrated Management of Emergency Vehicle Fleet, University of Maryland, College Park, MD, USA, 2006.

[23] A. Haghani and S. Yang, "Real-time emergency response fleet deployment: concepts, systems, simulation \& case studies," Dynamic Fleet Management, Springer, Boston, MA, USA, 2007.

[24] C. S. Lim, R. Mamat, and T. Braunl, "Impact of ambulance dispatch policies on performance of emergency medical services," IEEE Transactions on Intelligent Transportation Systems, vol. 12, no. 2, pp. 624-632, 2011.

[25] R. A. Garrido, P. Lamas, and F. J. Pino, "A stochastic programming approach for floods emergency logistics," Transportation Research Part E: Logistics and Transportation Review, vol. 75, no. 1, pp. 18-31, 2015.

[26] A. Moreno, D. Alem, and D. Ferreira, "Heuristic approaches for the multiperiod location-transportation problem with reuse of vehicles in emergency logistics," Computers \& Operations Research, vol. 69, no. 12, pp. 79-96, 2016.

[27] T. Ünlüyurt and Y. Tunçer, "Estimating the performance of emergency medical service location models via discrete event simulation," Computers \& Industrial Engineering, vol. 102, no. 4, pp. 467-475, 2016.

[28] B. Zhang, J. Peng, and S. Li, "Covering location problem of emergency service facilities in an uncertain environment," Applied Mathematical Modelling, vol. 51, no. 7, pp. 429-447, 2017.

[29] M. A. Akdogan, Z. P. Bayındır, and C. Iyigun, "Location analysis of emergency vehicles using an approximate queueing model," Transportation Research Procedia, vol. 22, no. 3, pp. 430-439, 2017.

[30] M. Vladimir, "Location models for emergency service applications," Informs Tutorials in Operations Research, vol. 174, no. 10, pp. 237-262, 2017.

[31] V. Bélanger, A. Ruiz, and P. Soriano, "Recent optimization models and trends in location, relocation, and dispatching of emergency medical vehicles," European Journal of Operational Research, vol. 272, no. 1, pp. 1-23, 2019.

[32] B. Zhang, H. Li, S. Li, and J. Peng, "Sustainable multi-depot emergency facilities location-routing problem with uncertain information," Applied Mathematics and Computation, vol. 333, no. 3, pp. 506-520, 2018.

[33] C. Lei, W.-H. Lin, and L. Miao, "A two-stage robust optimization approach for the mobile facility fleet sizing and routing problem under uncertainty," Computers \& Operations Research, vol. 67, no. 9, pp. 75-89, 2016.

[34] A. Atamturk and M. Zhang, "Two-stage robust network flow and design under demand uncertainty," Operations Research, vol. 55, no. 4, pp. 662-673, 2007.

[35] D. Bertsimas, E. Nasrabadi, and S. Stiller, "Robust and adaptive network flows," Operations Research, vol. 61, no. 5, pp. 1218-1242, 2013.

[36] L. V. Snyder, "Facility location under uncertainty: a review," IIE Transactions, vol. 38, no. 7, pp. 547-564, 2006. 


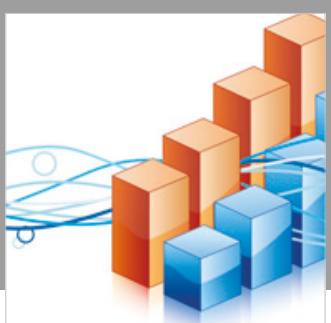

Advances in

Operations Research

\section{-n-m}
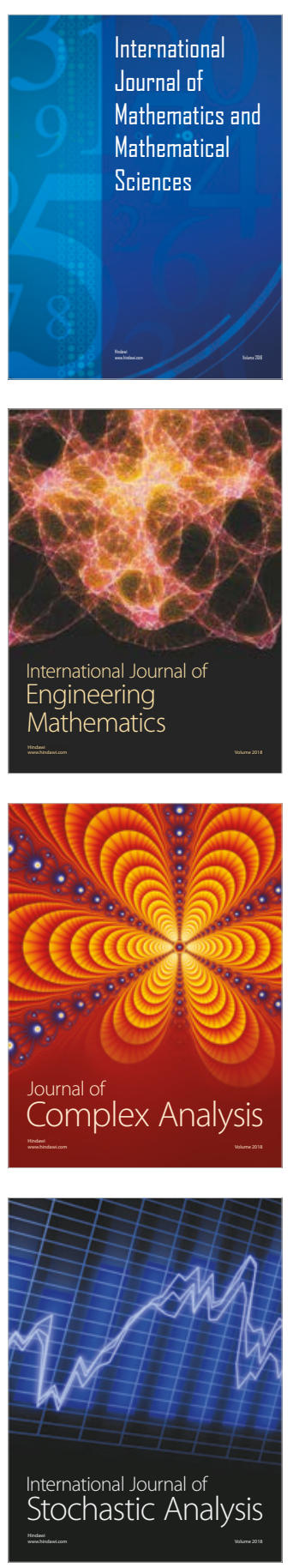
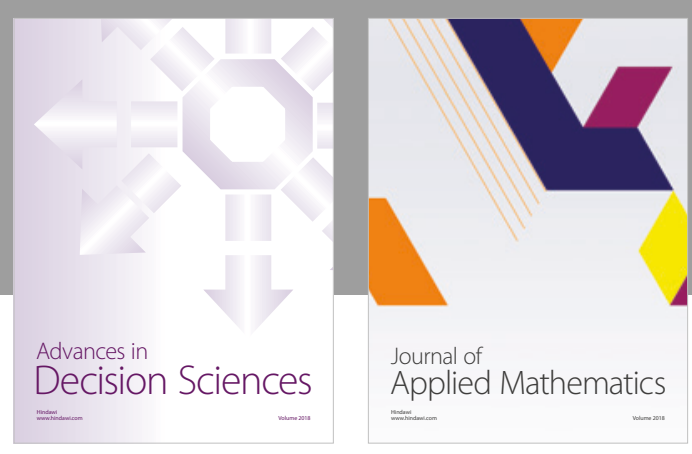

Journal of

Applied Mathematics
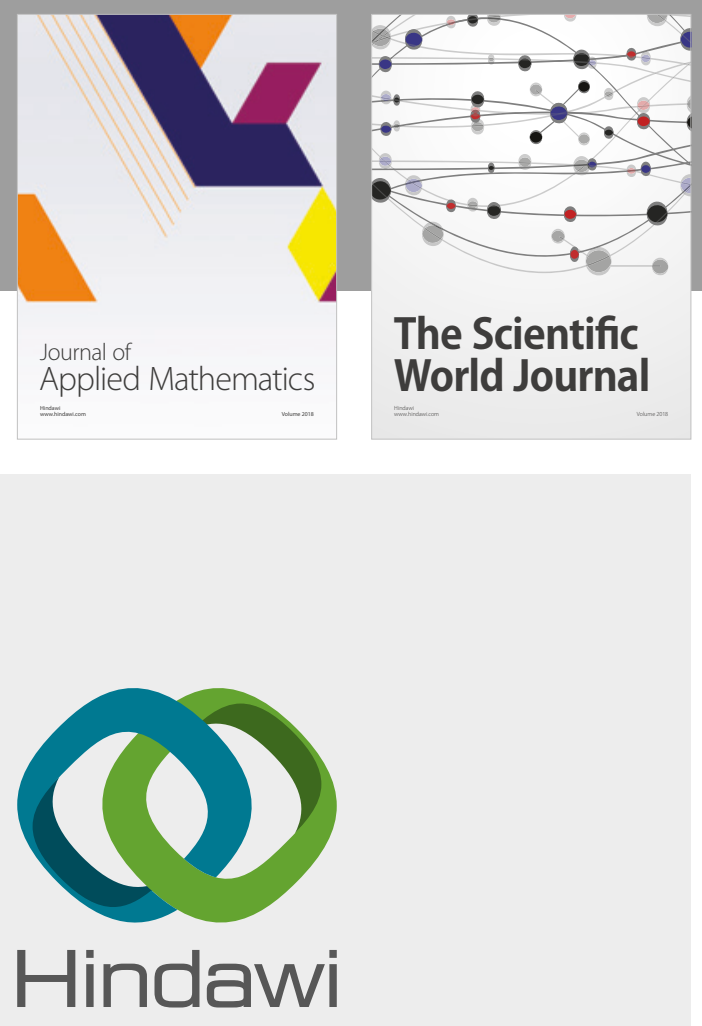

Submit your manuscripts at

www.hindawi.com

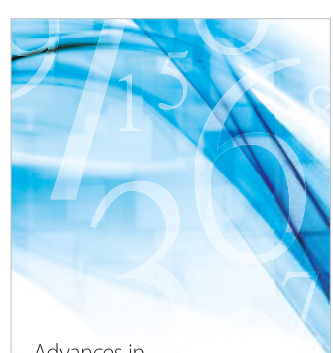

Advances in
Numerical Analysis
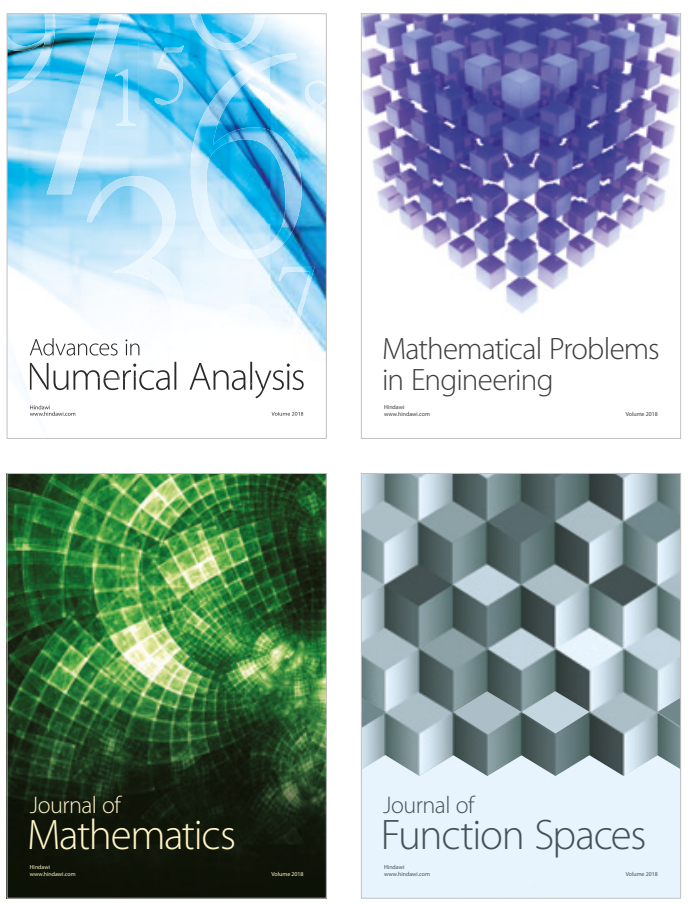

Mathematical Problems in Engineering

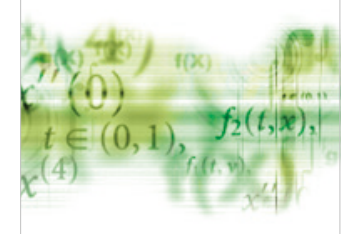

International Journal of

Differential Equations

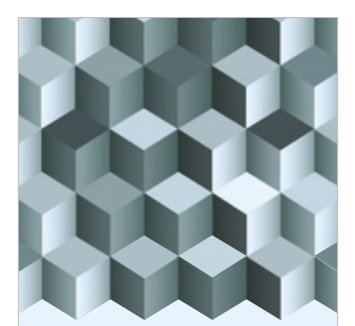

Journal of

Function Spaces
The Scientific

World Journal

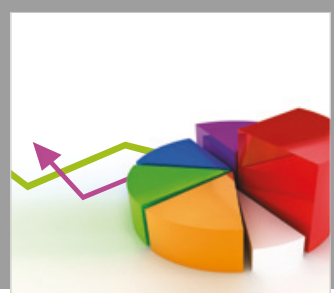

Journal of

Probability and Statistics
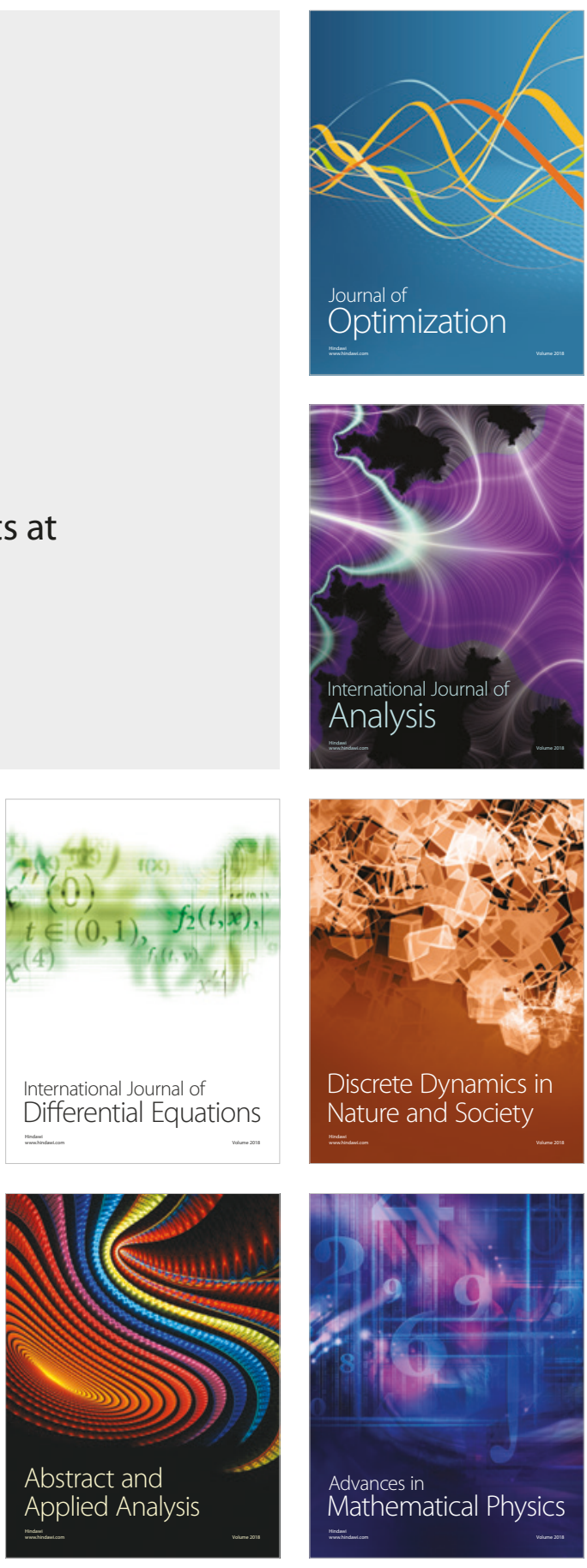